\title{
Respiratory symptoms in a patient with inflammatory bowel disease
}

\author{
Authors: Jennifer Capps ${ }^{A}$ and Mark Woodhead ${ }^{B}$
}
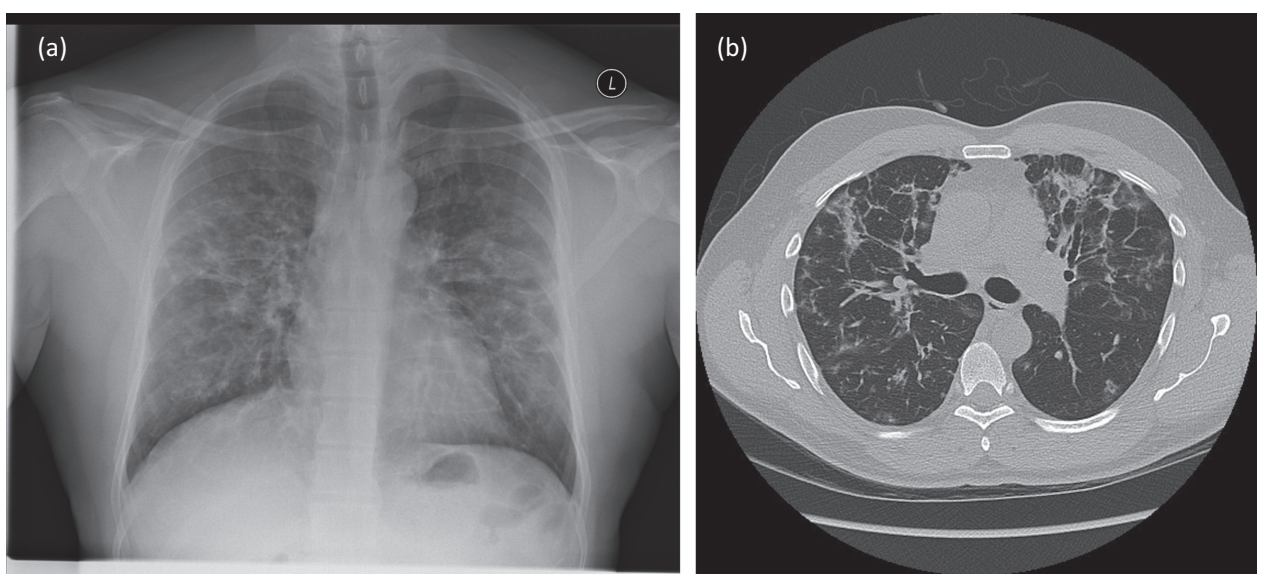

Fig 1. Baseline imaging (at presentation). (a) Chest X-ray. (b) CT thorax.
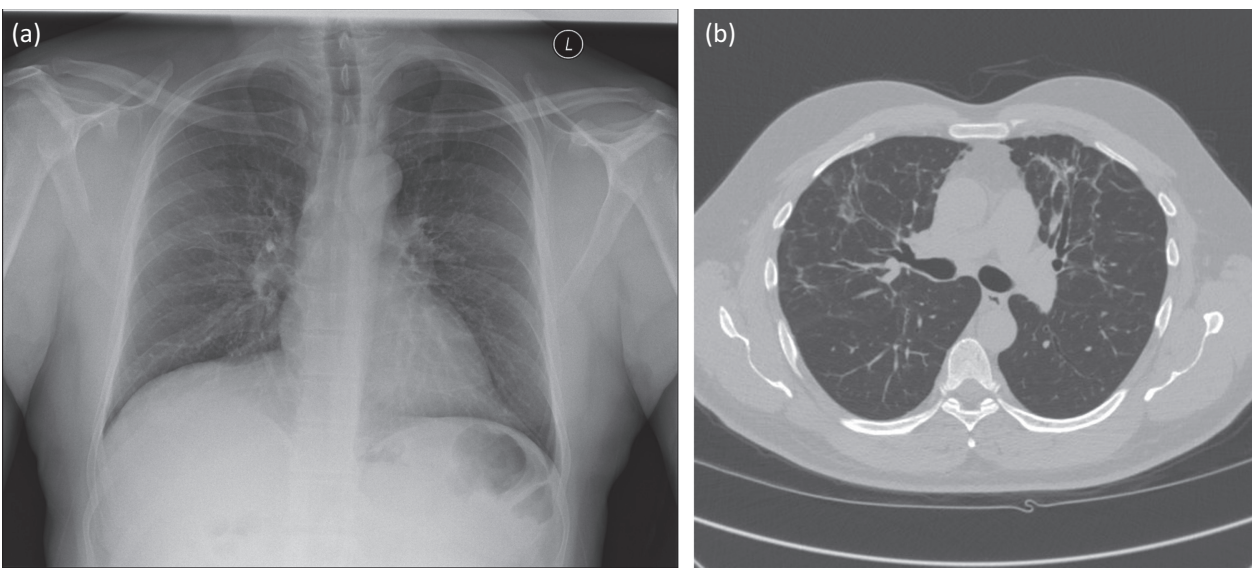

Fig 2. Images following completion of steroids. (a) Chest X-ray. (b) CT thorax.

A 47-year-old male presented with a 4-month history of breathlessness, chest discomfort and dry cough. He denied fevers, night sweats or weight loss. His medical history was of ulcerative

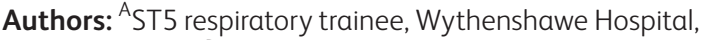
Manchester, UK; ${ }^{B}$ respiratory consultant and honorary clinical professor of respiratory medicine, Manchester Royal Infirmary, Manchester, UK colitis and hay fever. His current medications were azathioprine, mesalazine and infliximab (he had just received his third infusion). He had been screened and treated for latent tuberculosis (TB) prior to starting infliximab. He was a never smoker, an office worker, and kept no pets. He had no known family history, and was born in Pakistan. His most recent travel was 5 years ago.

The only abnormal findings on thoracic examination were bi-basal crackles. Routine blood tests were unremarkable. Three sputum samples were smear and culture negative for TB. A chest $X$-ray 1 year earlier was normal. A repeat chest X-ray was performed (Fig 1a). 
Possible diagnoses considered at this stage were infection (including TB), lung disease related to ulcerative colitis, and druginduced interstitial lung disease.

A CT scan (Fig 1b) showed multilobar ground-glass consolidation with focal traction bronchiectasis, predominantly in upper and mid-zones, sparing the lung peripheries. There were also ground-glass foci within the bronchovascular bundles. There were small calcified lymph nodes in the mediastinum, but no lymphadenopathy elsewhere. A bronchoscopy with lavage was performed. Bronchoscopic appearances were normal and transbronchial biopsy from the lingula was normal. The lavage fluid was culture negative, and predominantly lymphocytic $(61 \%)$.

A diagnosis of infliximab-related lung disease was made. Features supporting this include the lack of infective symptoms, the sterile sputum and the time course of the disease. In a large cohort of patients with rheumatoid arthritis, infliximabrelated pneumonitis occurred after a mean of 2.8 infusions. ${ }^{1}$ The radiological appearances and lymphocytic bronchial lavage were in keeping with other reports of anti-TNF-related lung disease. ${ }^{2,3}$

The infliximab had been stopped shortly before respiratory review. Reports suggest the disease may resolve with cessation of the offending drug, but steroids are often given. ${ }^{2}$ In this case prednisolone $40 \mathrm{mg}$ was initiated, and the patient's symptoms resolved rapidly. Steroids were weaned over 5 months, and imaging following this (Fig 2a and 2b) showed resolution of most abnormalities, leaving scattered ground-glass foci and mild traction bronchiectasis.

\section{Consent}

Patient consent was obtained to write and publish this article.

\section{Acknowledgements}

Thanks to Dr Devinda Karunaratne, consultant radiologist at Manchester Royal Infirmary, Manchester, who supplied the images included in this submission and provided a thoracic radiology opinion regarding the findings.

\section{References}

1 Takeuchi T, Tatsuki Y, Nogami Y et al. Postmarketing surveillance of the safety profile of infliximab in 5000 Japanese patients with rheumatoid arthritis. Ann Rheum Dis 2008;67:189-94.

2 Lee H, Jo K, Shim TS et al. Six cases of lung injury following antitumour necrosis factor therapy for inflammatory bowel disease. J Crohns Colitis 2015;9:1053-7.

3 Weinstein EJ, Lykens MG. Pulmonary Complications Of Infliximab Therapy. Chest 2009;136:(4_MeetingAbstracts):35S.

Address for correspondence: Dr Jennifer Capps, Wythenshawe Hospital, Southmoor Rd, Wythenshawe, Manchester M23 9LT, UK.

Email: Jennifer.capps@doctors.org.uk 